\title{
Onicomicosis en paciente inmunocompetente por Aspergillus sección Candidi
}

\author{
Onychomycosis in immunocompetent patient by Aspergillus section Candidi
}

\author{
Graciela Mariel Carballo', Jimena Agostina Miranda', Alicia Arechavala², Carlos Consigli', Daniela Mariel Fidalgo'
}

\begin{abstract}
Resumen
Introducción: los hongos miceliales no dermatofitos (HMND) pueden causar un gran número de infecciones, entre ellas, la onicomicosis. Su implicancia como agentes patógenos y su significado clínico en esta afección, se encuentran sujetos al cumplimiento de los criterios de Walshe y Mary P. English. Presentamos un caso de onicomicosis causada por un hongo hifomiceto poco frecuente, del género Aspergillus sección Candidi, en una paciente inmunocompetente y su estrategia terapéutica. Materiales y métodos: se estudió una paciente inmunocompetente, de 43 años de edad, con lesión en uña de primer dedo del pie derecho, de 10 años de evolución. Se realizó toma de muestra para examen micológico. Resultados: el examen directo reveló la presencia de hifas hialinas irregulares, ramificadas y tabicadas, compatibles con HMND. En muestras seriadas, desarrollaron colonias blanco algodonosas, cuya micromorfología fue concordante con Aspergillus sección Candidi. La identificación fue confirmada en la Unidad de Micología del Hospital de Infecciosas Francisco Javier Muñiz de la Ciudad Autónoma de Buenos Aires, Argentina. Allí también se realizaron pruebas de sensibilidad a antifúngicos, resultando sensible a itraconazol y terbinafina. Conclusión: exponemos un caso de onicomicosis causada por Aspergillus sección Candidi, HMND no queratinolítico, de escasa frecuencia de aparición, siendo el primer caso descripto y publicado en Argentina. También se planteó una estrategia terapéutica efectiva, que condujera a la cura clínica y microbiológica, de la uña de la paciente.
\end{abstract}

Palabras clave: hongos; Aspergillus; Candidi; onicomicosis; hialohifomicosis; inmunocompetente.

\begin{abstract}
Introduction: non-dermatophyte mycelial fungi (HMND) can cause a large number of infections, including onychomycosis. Its implication as pathogens and its clinical significance in this condition are subject to compliance with Walshe's and Mary P. English's criteria. We present a case of onychomycosis caused by hyphomycete rare fungus, of the genus Aspergillus section Candidi, in an immunocompetent patient and its therapeutic strategy. Materials and methods: a 43-year-old immunocompetent patient with a 10-year-old right-toe nail lesion was studied. Samples were taken for mycological examination. Results: the direct examination revealed the presence of irregular, branched and tabulated hyaline hyphae, compatible with HMND. In serial samples, they developed white cotton colonies, whose micromorphology was consistent with Aspergillus, section Candidi. The identification was confirmed at the Mycology Unit of Francisco Javier Muñiz Infectious Hospital in the Autonomous City of Buenos Aires, Argentina. Susceptibility to antifungals was also tested there, resulting in susceptibility to itraconazole and terbinafine. Conclusion: we present a case of onychomycosis caused by Aspergillus section Candidi, non-keratinolytic HMND, of rare occurrence, being the first case described and published in Argentina. An effective therapeutic strategy was also proposed that led to the patient's toenail's clinical and microbiological cure.
\end{abstract}

Keywords: fungi; Aspergillus; Candidi; onychomycosis; hyalohyphomycosis; immunocompetent.

Fecha de envío: 9 de enero de 2020 - Fecha de aceptación: 24 de agosto de 2020

\section{Introducción}

Se define como onicomicosis a la infección de uñas causada por levaduras y hongos filamentosos, principalmente dermatofitos, responsables del más del $90 \%$ de las mismas. Sin embargo, los hongos miceliales no dermatofitos (HMND), ante ciertas circunstancias, también pueden causar onicomicosis a pesar de su menor

(1) Nacional de Clínicas. Facultad de Ciencias Médicas. Córdoba. Argentina.

(2) Unidad de Micología. Hospital de Infecciosas Francisco Javier Muñiz. Buenos Aires. Argentina.

Autora de correspondencia: bestgmc@gmail.com 
capacidad queratinolítica (Negroni, 2008). Su incidencia es variable, pero un estudio multicéntrico realizado en Argentina revela que el 19\% de las onicomicosis son causadas por hongos anemófilos; el género Aspergillus es el tercero más aislado en orden de frecuencia (Relloso et al., 2012). El rol patógeno de los HMND ha sido muy discutido, ya que pueden comportarse como contaminantes, colonizantes o patógenos (Negroni R, 2008). Los criterios enunciados por Walshe y Mary P. English (Walshe \& English, 1966; English, 1976) siguen siendo fundamentales y aceptados universalmente para establecer el diagnóstico de onicomicosis por HMND. Estos son: recolección seriada de muestras a analizar (al menos tres), observación de elementos filamentosos en el examen directo y desarrollo de colonias abundantes y de la misma especie fúngica en los sitios de siembra.

Las especies del género Aspergillus, hongos miceliales hialinos, son de distribución universal, ampliamente diseminadas en el ambiente; se reproducen con facilidad y están presentes en suelo, agua, plantas y materia orgánica en descomposición. El extendido desarrollo ambiental de estos hongos, es favorecido por la falta de exigencias en las condiciones abióticas para el crecimiento y la eficiente dispersión de los conidios en el aire, lo que permite su sobrevivencia y diseminación aún en condiciones adversas (Shah \& Hazen, 2013).

Pueden producir gran cantidad de afecciones clínicas, de gravedad muy variable. Sin embargo, esto depende estrechamente de la respuesta inmune del hospedero; por ello Aspergillus es considerado un patógeno oportunista (Marr et al., 2002). Los complejos de especies comúnmente involucradas en la infección de uñas son: Aspergillus flavus, Aspergillus níger y Aspergillus terreus, entre otros. Aspergillus sección Candidi rara vez ha sido reportado como agente causal de onicomicosis y hasta el momento no se han publicado casos en Argentina (Nouripour-Sisakht et al., 2015; Michelli, 2016).

\section{Objetivo}

Presentar un caso clínico de onicomicosis de desafío diagnóstico, causado por un microorganismo poco frecuente, en paciente inmunocompetente.

\section{Caso clínico}

Paciente de sexo femenino, de 43 años de edad, ama de casa, procedente de zona rural de la provincia de Córdoba, Argentina. Consultó por lesión en uña de primer dedo del pie derecho, de 10 años de evolución. Al examen físico presentó compromiso del $70 \%$ de la lámina ungueal: leuconiquia subungueal, hiperqueratosis distal con paroniquia disto-lateral (Figura 1). Antecedentes patológicos: migraña, sobrepeso, hipotiroidismo subclínico y dislipemia.
Antecedentes tóxicos: tabaquismo (más de 20 cigarrillos diarios). Se realizó toma de muestra para examen micológico.

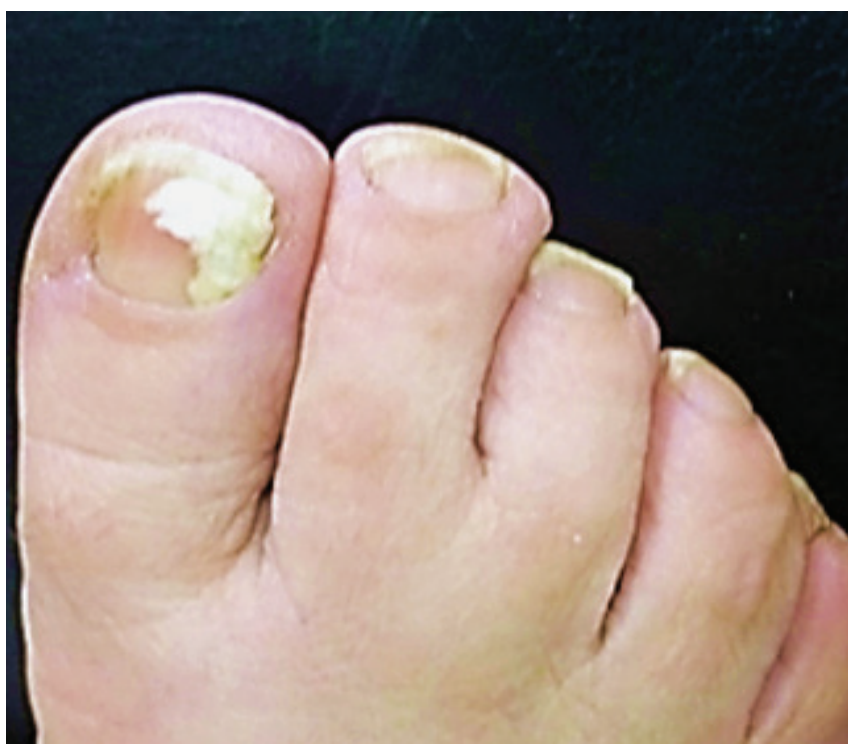

Figura 1: Macrofotografía de primera uña de pie derecho postratamiento: placa ungueal libre de lesión.

\section{Micológico directo y cultivo de uña de pie}

El examen micológico directo de la placa ungueal reveló presencia de hifas hialinas irregulares, ramificadas y tabicadas, compatibles con HMND. Los cultivos se realizaron por duplicado en agar Sabouraud glucosado (ASG) con antibióticos; fueron incubados durante 2 semanas a temperatura ambiente. En todos los puntos de siembra, hubo desarrollo de colonias color blanco, inicialmente de aspecto granuloso y luego algodonoso (Figura 2). Por las características macro y microscópicas, fue identificado un hongo hialohifomicete, perteneciente al género Aspergillus spp. Se realizó microcultivo en agar papa (AP). Microscópicamente se observó en detalle: conidióforos hialinos de pared gruesa y cabeza aspergilar redondeada rodeada por métulas biseriadas. De las mismas, nacían cadenas de conidios hialinos, globosos y de pared fina, compatibles con Aspergillus sección Candidi (Figura 3). La paciente fue citada nuevamente para repetir el estudio micológico, siguiendo los criterios de Walshe y Mary P. English, obteniéndose idénticos resultados.

La confirmación de la identificación de la cepa se realizó por observación macro y micromorfológica en la Unidad de Micología del Hospital de Infecciosas Francisco Javier Muñiz de la ciudad autónoma de Buenos Aires, Argentina. Allí también se realizaron pruebas de sensibilidad antifúngica (SA) por difusión en agar, empleando tabletas Neo Sensitabs Rosco ${ }^{\circledR}$ (Rosco Diagnóstica A/S, 30, DK-2630, Dinamarca) según documento M 51-A (CLSI, 
2010) del Instituto de Estándares Clínicos y de Laboratorio (CLSI); resultando sensible para itraconazol $(37 \mathrm{~mm})$ y terbinafina $(50 \mathrm{~mm})$. La concentración inhibitoria mínima (CIM) utilizando método de microdilución en caldo, según documento M38 3er ed. del CLSI (CLSI, 2017), fue practicada para itraconazol: $0,125 \mathrm{ug} / \mathrm{ml}$, y terbinafina: $0,06 \mathrm{ug} / \mathrm{ml}$ (ambos sensibles).

Con estos resultados y siguiendo las recomendaciones de Negroni (Negroni, 2008), se indicó tratamiento por vía oral con itraconazol $400 \mathrm{mg}$ /día una semana al mes durante 2 meses, y luego terbinafina 500 mg/día una semana al mes durante 2 meses más. Se hicieron controles de la función hepática y renal al comenzar y concluir el mismo; se utilizó además amorolfina 5\% (laca) 2 veces por semana como coadyuvante tópico.

Se realizó un nuevo estudio micológico 8 meses postratamiento sistémico, obteniéndose cultivo de hongos negativo. A modo preventivo se continuó con medicación tópica durante 6 meses adicionales; a los 30 días de suspendida la misma, se repitió el cultivo con idéntico resultado al anterior, confirmando así la cura clínica y micológica de la uña (Figura 4).

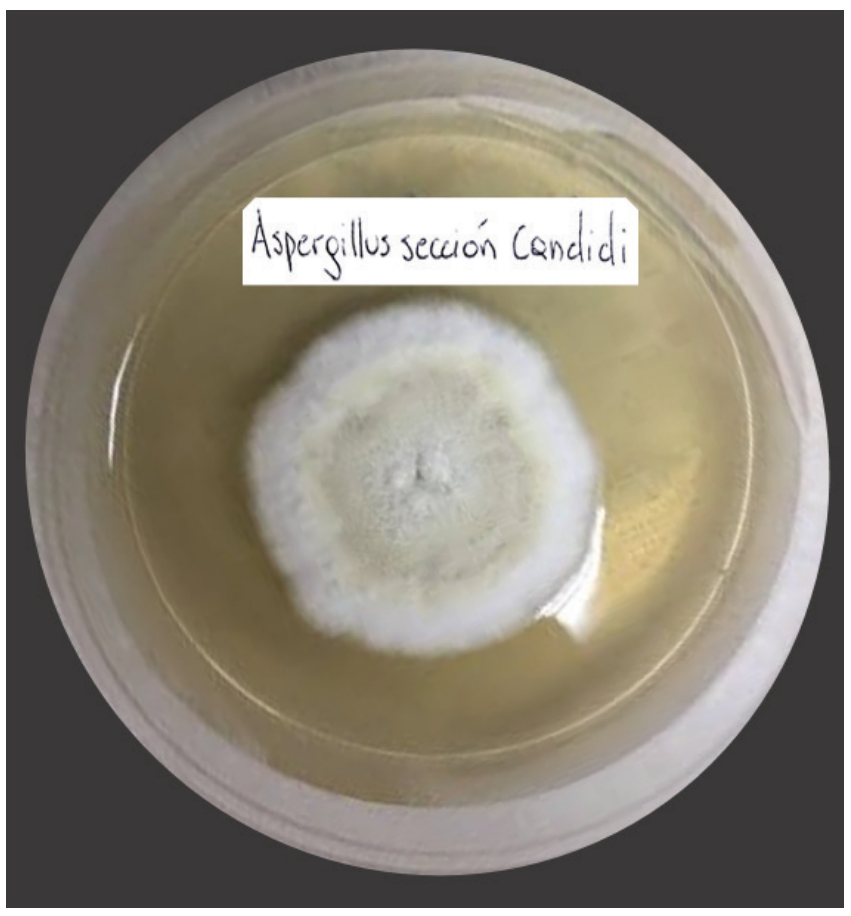

Figura 2: Cultivo de Aspergillus sección Candidi en agar Sabouraud glucosado. colonias color blanco de aspecto granuloso algodonoso.

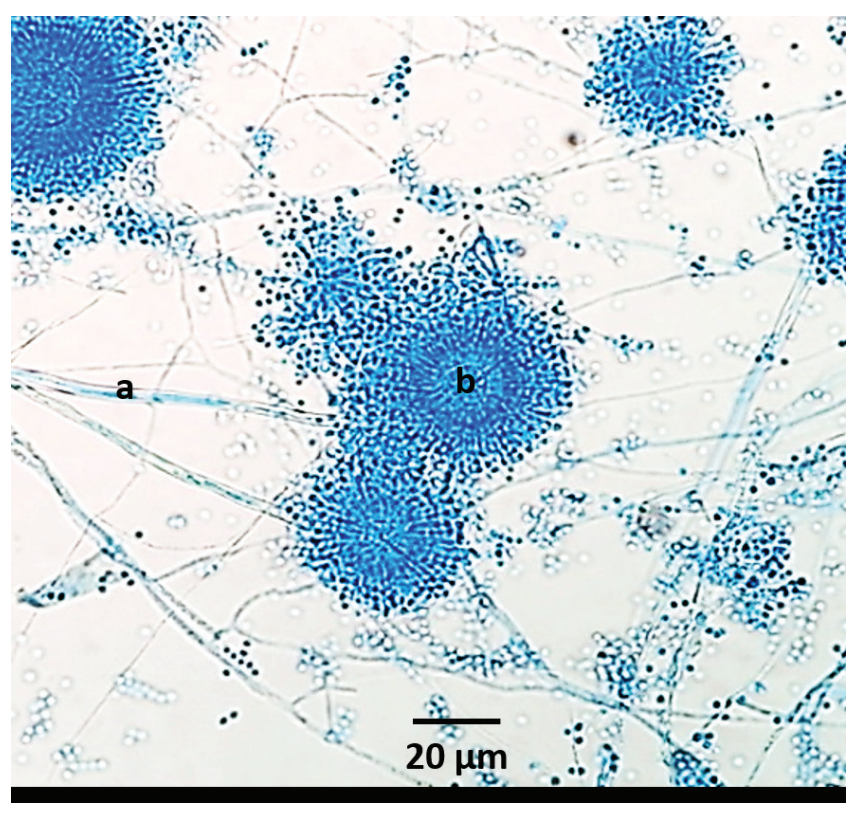

Figura 3: Aspecto microscópico de colonia de Aspergillus sección Candidi en agar papa. Conidióforos hialinos de pared gruesa (a), cabeza aspergilar rodeada por métulas biseriadas con cadenas de conidios hialinos, globosos, de pared fina (b) (Azul de lactofenol-1000x).

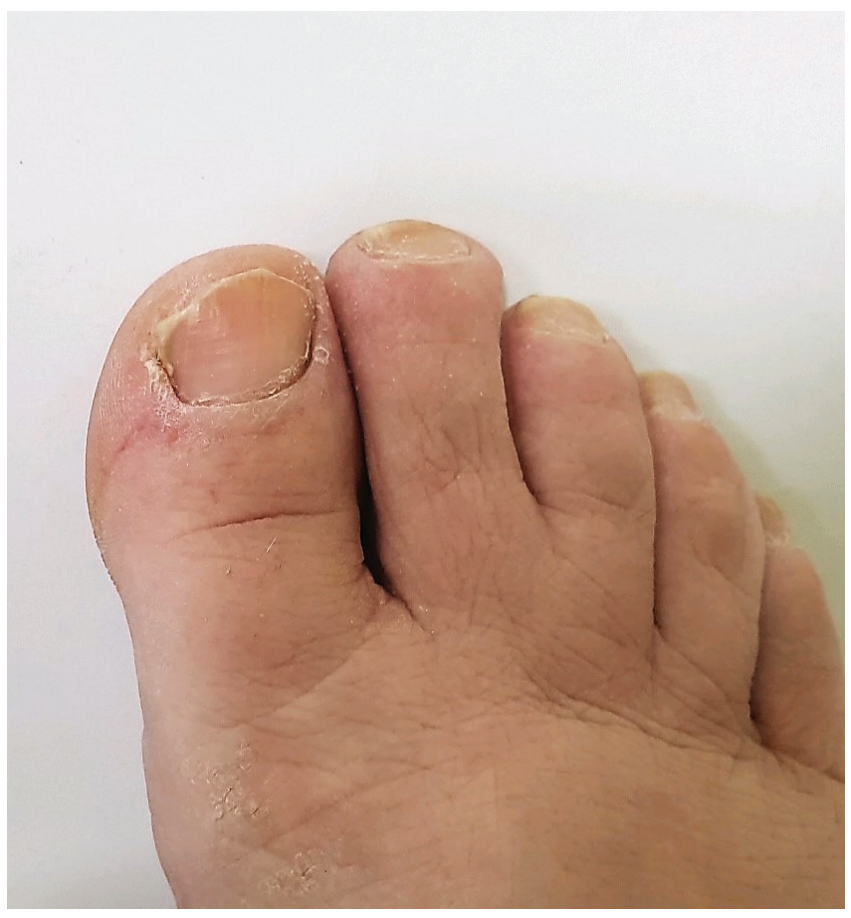

Figura 4: Onicomicosis. leuconiquia subungueal, hiperqueratosis distal con paroniquia disto-lateral de uña de primer dedo del pie derecho. 


\section{Discusión}

Las onicomicosis causadas por especies del género Aspergillus ocupan el tercer lugar en nuestro país, se aíslan fundamentalmente de uñas de pie y Aspergillus candidus, especie de la sección Candidi, está ampliamente distribuido en la naturaleza (Negroni, 2008; Relloso et al., 2012). Se desarrolla en vegetales en estado de descomposición y es la especie de hongo más frecuentemente hallada como contaminante de granos de cereales y harinas. Hasta la fecha, ha sido reportado en casos de aspergilosis invasiva, otomicosis y granuloma cerebral; raramente afecta las uñas en el ser humano, comunicándose pocos casos en el mundo. (Del Palacio et al., 2001; Marr et al., 2002; Ahmadi et al., 2012).

El hallazgo excepcional de este hongo como agente causal de onicomicosis y su clínica similar a la causada por dermatofitos hace difícil su diagnóstico, con el agravante de no responder a los tratamientos convencionales (Llambrich \& Lecha, 2002; Nouripour-Sisakht et al., 2015).

En nuestro caso, el aislamiento del hongo en muestras seriadas de uñas, y ante ausencia de crecimiento de dermatofitos, confirmó su rol etiológico (Walshe \& English, 1966; English, 1976).

La confirmación diagnóstica del hongo se realizó según criterios macro y micromorfológicos, en el laboratorio de referencia: Unidad de Micología del Hospital de Infecciosas Francisco Javier Muñiz de la ciudad Autónoma de Buenos Aires, Argentina. Si bien los métodos de identificación definitivos se basan en métodos polifásicos (morfológicos, fisiológicos, moleculares, y ecológicos), que permiten confirmar la identidad taxonómica del microorganismo (Houbraken et al., 2014), estas herramientas no fueron factibles de aplicar en nuestro caso. Además, debemos aclarar que Aspergillus candidus sensu lato era, hasta hace poco, la única especie reconocida dentro de la sección Candidi; sin embargo, un grupo de investigadores publicó recientemente que este hongo conforma un complejo de especies (Michelli, 2016; Hubka et al., 2018).

No existen aún puntos de cortes, publicados por el CLSI, respecto a los antifúngicos más efectivos para utilizar en el tratamiento del hongo aquí presentado; sin embargo, los resultados de las pruebas de sensibilidad con discos y la microdilución en placa (CIM), fueron concluyentes con lo publicado por prestigiosos autores (Espinel et al., 2010). Ha sido ensayada la asociación itraconazol y terbinafina, ambas por vía oral. Los estudios in vitro han demostrado que la misma es sinérgica frente a dermatofitos y HMND (Negroni, 2008); se recomienda la administración de estos fármacos en forma secuencial y en pulso, mejorando las tasas de curación y reduciendo los efectos adversos ( Gupta \& Ryder, 2003; Negroni, 2008; Carillo et al., 2010).
Si bien no hay consenso sobre cuál es la terapéutica más efectiva para las onicomicosis por HMND, muchos autores afirman que el tratamiento combinando vía oral y tópica, es el que ofrece mejores resultados (Negroni, 2008; Fallas \& Zumbado, 2014). En nuestra paciente, el uso de itraconazol más terbinafina combinado con amorolfina al $5 \%$ en laca fue exitoso.

En definitiva, describimos un caso de onicomicosis en una paciente femenina inmunocompetente, causada por un hongo del género Aspergillus sección Candidi, HMND no queratinolítico, de escasa frecuencia de aparición, siendo el primer caso descripto y publicado en Argentina. También se planteó una estrategia terapéutica efectiva que condujo a la cura clínica y microbiológica de la paciente.

\section{Contribuciones y reconocimientos}

Carballo Graciela Mariel: diagnóstico micológico, concepción y diseño del trabajo, revisión de la literatura, redacción de manuscrito, aprobación de versión final. Miranda Jimena Agostina: redacción de manuscrito, aprobación de versión final. Fidalgo Daniela Mariel: aporte de paciente, concepción y diseño de trabajo, revisión de la literatura, aprobación de versión final. Consigli Carlos: revisión crítica de manuscrito y aprobación de versión final. Arechavala Alicia: confirmación de identificación y sensibilidad antifúngica con tabletas y CIM, revisión de la literatura, aprobación de versión final. Fuente de financiamiento: ninguna que declarar. Conflicto de interés: ninguno que declarar.

\section{Bibliografía}

Ahmadi B, Hashemi SJ, Zaini F, Shidfar MR, Moazeni M, Mousavi B, Noorbakhsh F, Gheramishoar M, Hossein Pour L \& Rezaie S. (2012). A case of onychomycosis caused by Aspergillus candidus. Medical Mycology Case Reports 1, 45-48.

Carrillo-Muñoz AJ, Tur-Tur C, Hernández-Molina JM, Santos P, Cárdenes D \& Giusiano G. (2010). Antifúngicos disponibles para el tratamiento de las micosis ungueales. Revista Iberoamericana de Micología 27, 49-56

CLSI. (2010). Method for Antifungal Disk Diffusion Susceptibility Testing of Nondermatophyte Filamentous Fungi; Approved Guideline. CLSI document M51-A. Wayne, PA:Clinical and Laboratory Standards Institute.

CLSI. (2017). Reference Method for Broth Dilution Antifungal Susceptibility Testing of Filamentous Fungi, 3rd ed. CLSI standard M38. Wayne, PA: Clinical and Laboratory Standards Institute.

Del Palacio A, Pazos C \& Cuetara S. (2001). Onicomicosis por hongos filamentosos no dermatofitos. Enfermedades Infecciosas y Microbiología Clínica 19, 439-42. 
English MP. (1976). Nails and fungi. British Journal of Dermatology: BJD 94, 697-701.

Espinel-Ingroff A, Diekema DJ, Fothergill A, Johnson E, Pelaez T, Pfaller MA, Rinaldi MG, Canton E \& Turnidge J. (2010). Wild-type MIC distributions and epidemiological cutoff values for the triazoles and six Aspergillus spp. for the CLSI broth microdilution method (M38-A2 document). Journal of Clinical Microbiology: JCM 48, 3251-7.

Fallas MU \& Zumbado Salazar CA. (2014). Onicomicosis causadas por hongos miceliales no dermatofitos. Revista Médica de Costa Rica y Centroamérica 71, 733 - 736.

Gupta AK \& Ryder JE. (2003). How to improve cure rates for the management of onychomycosis. Dermatologic Clinics 21, 499- 505.

Houbraken J, de Vries RP \& Samson RA. (2014). Modern taxonomy of biotechnologically important Aspergillus and Penicillium species. Advances in Applied Microbiology 86, 199-249.

Hubka V, Nováková A, Jurjević Ž, Sklenář F, Frisvad JC, Houbraken J, Arendrup MC, Jørgensen KM, Siqueira JPZ, Gené J \& Kolařík M. (2018). Polyphasic data support the splitting of Aspergillus candidus into two species; proposal of Aspergillus dobrogensis sp. nov. International Journal of Systematic and Evolutionary Microbiology 68, 995-1011.

Llambrich A \& Lecha M. (2002). Tratamiento actual de las onicomicosis. Revista Iberoamericana de Micología 19, 127-129.
Marr KA, Patterson T \& Denning D. (2002). Aspergillosis. Pathogenesis, clinical manifestations, and therapy. Infectious Disease Clinics of North America 16, 875-94.

Michelli P. (2016). Aspergillus y sus complejos de especies. En Hongos oportunistas levaduriformes y filamentosos comunes en la clínica. Manual de laboratorio, eds. Giusiano GE \& Piontelli E, pp. 48-85. Consejo Nacional de Investigaciones Científicas y Técnicas, Resistencia, Argentina. Autopublicado.

Negroni R. (2008). Tratamiento de las onicomicosis. Revista de Patología Tropical 37, 89-109.

Nouripour-Sisakht S, Mirhendi H, Shidfar MR, Ahmadi B, Rezaei-Matehkolaei A, Geramishoar M, Zarei F \& Jalalizand N. (2015). Aspergillus species as emerging causative agents of onychomycosis. Journal of Medical Mycology: JMM 25, 101-7.

Relloso S, Arechavala A, Guelfand L, Maldonado I, Walker L, Agorio I, Reyes S, Giusiano G, Rojas F, Flores V, Capece P, Posse G, Nicola F, Tutzer S \& Bianchi M. (2012). Onicomicosis: estudio clínico, epidemiológico y micológico multicéntrico. Revista lberoamericana de Micología 29, 157-63.

Shah AA \& Hazen KC. (2013). Diagnostic accuracy of histopathologic and cytopathologic examination of Aspergillus species. American Journal of Clinical Pathology: AJCP 139, 55-61.

Walshe MM \& English MP. (1966). Fungi in nails. British Journal of Dermatology: BJD 78, 198-207. 